\title{
On Einstein clusters as galactic dark matter halos
}

\author{
C. G. Böhmer ${ }^{1 \star}$ and T. Harko ${ }^{2}$ \\ ${ }^{1}$ Institute of Cosmology $\&$ Gravitation, University of Portsmouth, Portsmouth PO1 2EG, UK \\ ${ }^{2}$ Department of Physics and Center for Theoretical and Computational Physics, The University of Hong Kong, \\ Pok Fu Lam Road, Hong Kong
}

\begin{abstract}
We consider global and gravitational lensing properties of the recently suggested Einstein clusters of WIMPs as galactic dark matter halos. Being tangential pressure dominated, Einstein clusters are strongly anisotropic systems which can describe any galactic rotation curve by specifying the anisotropy. Due to this property, Einstein clusters may be considered as dark matter candidates. We analyse the stability of the Einstein clusters against both radial and non-radial pulsations, and we show that the Einstein clusters are dynamically stable. With the use of the Buchdahl type inequalities for anisotropic bodies, we derive upper limits on the velocity of the particles defining the cluster. These limits are consistent with those obtained from stability considerations. The study of light deflection shows that the gravitational lensing effect is slightly smaller for the Einstein clusters, as compared to the singular isothermal density sphere model for dark matter. Therefore lensing observations may discriminate, at least in principle, between Einstein cluster and other dark matter models.
\end{abstract}

Key words: Einstein clusters - dark matter - WIMPs.

\section{INTRODUCTION}

In 1939 Einstein (Einstein 1939) presented a model of a thick spherical shell composed of test particles of equal mass, each moving in a circular geodesic orbit in the field of all the other. Such a system has spherical symmetry and may be used to model globular clusters of stars, since it gives a good approximation to the average density of matter and the average value of the gravitational field.

Einstein clusters have been studied extensively by Gilbert (1954); Hogan (1973); Baneriee and Som (1981); Comer and Katz (1993); Comer et al. (1993), and the main physical properties of the cluster have been derived. In particular, the energy-momentum tensor components $T_{i}^{j}$ for the cluster have been obtained in several representations. Due to the spherical symmetry, the only non-vanishing components of $T_{i}^{j}$ are $T_{t}^{t}=\rho^{(\mathrm{eff})}, T_{r}^{r}=-p_{r}^{\text {(eff) }}$ and $T_{\theta}^{\theta}=T_{\varphi}^{\varphi}=-p_{\perp}^{(\mathrm{eff})}$, where $\rho^{(\text {eff })}$ is the effective energy-density of the cluster and $p_{r}^{\text {(eff) }}$ and $p_{\perp}^{\text {(eff) }}$ are the radial and tangential pressures, respectively. Since the junction conditions require $p_{r}^{(\mathrm{eff})}$ to be continuous across the boundary of of each layer of the shell, it follows that for the Einstein cluster $p_{r}^{(\text {eff })}=0$. The nonvanishing energy-momentum components can be expressed in terms of the velocity of the particles in the cluster and the density only. It can also be interpreted as giving rise to rotation without introducing global angular momentum.

\footnotetext{
* E-mail: christian.boehmer@port.ac.uk; harko@hkucc.hku.hk
}

Therefore, the Einstein clusters are examples of spherically symmetric systems with an anisotropic energymomentum, for which the radial pressure is different from the tangential one, $p_{r}^{\text {(eff })} \neq p_{\perp}^{\text {(eff) }}$. Anisotropic spherically symmetric matter distributions have been extensively studied in the past, and several important physical characteristics of these systems have been obtained. In particular, several generalisations of the Buchdahl bound on the massradius ratio, the redshift and the value of the anisotropy parameter have been obtained, also in the presence of the cosmological constant by Böhmer and Harko (2006). Similarly to the case of isotropic systems (Böhmer and Harko $2005 \mathrm{~b}$ ), the presence of the cosmological constant also determines the existence of a minimal mass for anisotropic matter distributions.

An interesting physical application of the Einstein clusters has recently been suggested by Lake (2006). The mass of the Einstein cluster is proportional to the square of the tangential velocity and to the distance to the centre. This behaviour is very similar to that observed in the case of test particles in stable circular orbits around the galactic centre. In most galaxies, neutral hydrogen clouds are observed at large distances from the centre, much beyond the extent of the luminous matter, see Binney and Tremaine (1987); Persic et al. (1996). Since these clouds move in circular orbits with velocity $V(r)$, the orbits are maintained by the balance between the centrifugal acceleration $V(r)^{2} / r$ and the gravitational attraction force $G M(r) / r^{2}$ of the total mass $M(r)$ contained within the orbit. This allows us to express 
the mass profile of the galaxy in the form $M(r)=r V^{2} / G$. This mass profile can be obtained rigorously for the Einstein clusters. Observations show that the rotational velocities increase near the centre of the galaxy and then remain nearly constant at a value of $V_{0} \sim 200 \mathrm{~km} / \mathrm{s}$ Binnev and Tremaine 1987; Persic et al. 1996). Consequently, the mass within a distance $r$ from the centre of the galaxy increases linearly with $r$, even at large distances where very little luminous matter can be detected.

It is the purpose of the present analysis to show that the Einstein cluster may indeed serve as a physically acceptable dark matter model (Lake 2006), which satisfies all energy conditions, and is also in agreement with the generic results on anisotropic matter distributions, obtained in Böhmer and Harko (2006). As a first step we obtain the energy-momentum tensor for the Einstein cluster, and derive the mass-velocity relation in the presence of a cosmological constant. Next we show that the Einstein cluster is dynamically stable against radial and non-radial perturbations. Several Buchdahl type physical bounds for the Einstein cluster are also considered, leading to some upper bounds for the velocity of the particles in the cluster and to the estimation of its minimum mass.

An observational possibility of testing the idea of the dark matter as an Einstein cluster is the study of the light deflection and lensing. We derive the deflection angle of light in an Einstein cluster, and compare its value with the deflection angle in the standard isothermal sphere dark matter model. It is pointed out that both models can in principle be distinguished by observation.

The present paper is organised as follows. The energy momentum tensor for the Einstein cluster is derived in Section 2 The stability of the Einstein cluster is considered in Section 3 General bounds on the velocity and mass of the cluster are obtained in Section 4 and the lensing effect is considered in Section 5. We discuss and conclude our results in the final Section 6. Throughout this paper we use a system of units so that $c=1$.

\section{THE EINSTEIN CLUSTER MODEL}

We consider a system consisting of particles of rest mass $m$, each describing a circular orbit about the centre $O$ of the cluster, in the presence of a cosmological constant $\Lambda$. The effect of the collision between particles is neglected. Since the system is static and has spherical symmetry, the line element is given by

$$
d s^{2}=e^{\nu} d t^{2}-e^{\lambda} d r^{2}-r^{2}\left(d \theta^{2}+\sin ^{2} \theta d \varphi^{2}\right),
$$

where we have denoted the coordinates as $x^{0}=t, x^{1}=r$, $x^{2}=\theta$ and $x^{3}=\varphi$, respectively, $\nu$ and $\lambda$ are functions of $r$ only, and $r \leqslant R$, with $r=R$ being the boundary of the cluster. This metric is joined continuously at $r=R$ with the standard Schwarzschild - de Sitter metric, having the mass parameter $M$, which contains the mass of the galactic core and the mass of the dark matter halo.

The energy-momentum of the particles in the cluster is given by

$$
T_{i}^{j}=\rho\left\langle g_{i k} \frac{d x^{j}}{d s} \frac{d x^{k}}{d s}\right\rangle
$$

where $\rho$ is the energy density and \langle\rangle denotes the mean value of the energy and momentum of a particle for all the particles in the volume element around an arbitrary point $P$. In the following we consider the particles in the cluster moving in the plane $\varphi=$ constant. We define the components $v^{\alpha}$ of the three-velocity of the particles, measured in terms of the proper time, that is, by an observer located at the given point, by Landau and Lifshitz (1998)

$$
v^{\alpha}=\frac{d x^{\alpha}}{\sqrt{g_{t t}} d x^{t}} .
$$

The square of the velocity is given by $V^{2}=v_{\alpha} v^{\alpha}=$ $-g_{\alpha \beta} v^{\alpha} v^{\beta}$. Since the particles are in circular motion around the centre $O$ of the cluster, there is only one non-vanishing component of the three-velocity,

$$
v^{2}=v^{\theta}=e^{-\nu / 2} \frac{d \theta}{d t},
$$

with the magnitude of the velocity having the value

$$
V^{2}=r^{2} e^{-\nu}\left(\frac{d \theta}{d t}\right)^{2} .
$$

From Eq. (1) we immediately obtain

$$
e^{\nu}\left(\frac{d t}{d s}\right)^{2}=\left(1-V^{2}\right)^{-1}
$$

Then, by taking the mean value in Eq. (2) we obtain the components of the energy-momentum tensor of the Einstein cluster as

$$
\begin{aligned}
& T_{t}^{t}=\rho^{(\mathrm{eff})}=\rho\left(1-V^{2}\right)^{-1}, \quad T_{r}^{r}=-p_{r}^{(\mathrm{eff})}=0, \\
& T_{\theta}^{\theta}=T_{\varphi}^{\varphi}=-p_{\perp}^{(\mathrm{eff})}=-\frac{1}{2} \rho V^{2}\left(1-V^{2}\right)^{-1} .
\end{aligned}
$$

Anisotropic matter distributions may be described in terms of the anisotropy parameter $\Delta=p_{\perp}^{\text {(eff) }}-p_{r}^{\text {(eff) }}$, which for the Einstein cluster takes the form

$$
\Delta=p_{\perp}^{(\mathrm{eff})}=\frac{1}{2} \rho V^{2}\left(1-V^{2}\right)^{-1} .
$$

If $\Delta>0, \forall r \neq 0$, as is the case for the Einstein cluster, the body is tangential pressure dominated.

In the case of an anisotropic system with vanishing radial pressure the condition of the conservation of the energymomentum tensor, $\nabla_{j} T^{i j}=0$ gives

$$
\nu^{\prime}=\frac{4 \Delta}{\rho^{(\mathrm{eff})} r},
$$

where the prime denotes differentiation with respect to $r$, so that for the Einstein clusters we obtain

$$
\nu^{\prime}=\frac{2}{r} V^{2} .
$$

In static and spherically symmetric spacetimes the field equations reduce to three independent equations, which imply conservation of energy-momentum. Since in the present discussion it is convenient to use the conservation equation directly, it suffices to consider the first two Einstein field equations, which are

$$
\begin{aligned}
-e^{-\lambda} & \left(\frac{1}{r^{2}}-\frac{\lambda^{\prime}}{r}\right)+\frac{1}{r^{2}}=8 \pi G \rho\left(1-V^{2}\right)^{-1}+\Lambda, \\
e^{-\lambda}\left(\frac{\nu^{\prime}}{r}+\frac{1}{r^{2}}\right)-\frac{1}{r^{2}} & =-\Lambda,
\end{aligned}
$$


respectively.

The latter Eq. (13) leads to

$$
e^{-\lambda}=\left(1-\Lambda r^{2}\right)\left(2 V^{2}+1\right)^{-1},
$$

whereas from Eq. (12) we obtain

$$
e^{-\lambda}=1-\frac{2 G m(r)}{r}-\frac{\Lambda}{3} r^{2}
$$

where we have defined the mass inside the radius $r$ by

$$
m(r)=4 \pi \int_{0}^{r} \rho\left(1-V^{2}\right)^{-1} r^{\prime 2} d r^{\prime} .
$$

The metric function $\lambda$ can be eliminated from Eqs. (14) and (15) to obtain the explicit dependence of $m(r)$ on $V^{2}$ and $r$ as

$$
\frac{2 G m(r)}{r}=\frac{2 V^{2}+\Lambda r^{2}}{1+2 V^{2}}-\frac{\Lambda}{3} r^{2} .
$$

Hence, the explicit dependence of the velocity on the mass of the cluster is

$$
V^{2}=\left(\frac{G m(r)}{r}-\frac{\Lambda}{3} r^{2}\right)\left(1-\frac{2 G m(r)}{r}-\frac{\Lambda}{3} r^{2}\right)^{-1} .
$$

For the case of low rotational velocities $V^{2} \ll 1$ (the Newtonian limit) the expression for the tangential velocity becomes

$$
V^{2}=\frac{G m(r)}{r}-\frac{\Lambda}{3} r^{2}
$$

In the same approximation the metric tensor coefficient $e^{\nu}$ is given by

$$
e^{\nu(r)}=\exp \left[2 \int\left(\frac{G m\left(r^{\prime}\right)}{r^{\prime 2}}-\frac{\Lambda}{3} r^{\prime}\right) d r^{\prime}\right] .
$$

In the Einstein cluster interpretation of the dark matter, the tangential velocity tends to a constant value for large radii $\lim _{r \rightarrow \infty} V(r)=V_{0}=$ constant. Therefore in this region the density of the Einstein cluster is given by

$$
\rho(r)=\frac{V_{0}^{2}}{4 \pi G r^{2}}+\frac{\Lambda}{4 \pi G} .
$$

\section{STABILITY OF THE EINSTEIN CLUSTERS AGAINST RADIAL AND NON-RADIAL PERTURBATIONS}

The issue of stability is of great importance in the study of Newtonian and general relativistic models of self-gravitating objects. Its relevance becomes evident if we recall that any astrophysical model is physically uninteresting if it is unstable against small perturbations, and different degrees of stability/instability will lead to different patterns of evolution in the collapse of self gravitating objects. Therefore, as realistic dark matter models the Einstein clusters must be dynamically stable.

The stability of astrophysical objects has been the subject of many investigations. Most studies, however, assume that the matter is described by an isotropic perfect fluid. On the other hand one expects that the anisotropy will change the evolution of self-gravitating systems Hillebrandt and Steinmetz (1976); Herrera and Santos (1997).

In the present Section we analyse the stability of the
Einstein clusters under small radial and non-radial perturbations. In both cases we neglect the effect of the cosmological constant since its effects on the dynamical stability small for the present model, see e.g. Böhmer and Harko (2005a); Hledik et al. (2007).

\subsection{Stability against radial perturbations}

We assume that initially the equilibrium configuration of the Einstein cluster is spherically symmetric, and that the perturbations preserve this symmetry. Under these perturbations only radial motions will ensue. Hence the metric of the spacetime is still given by Eq. (1), but with $\nu$ and $\lambda$ functions of both $t$ and $r, \nu=\nu(t, r), \lambda=$ $\lambda(t, r)$. If we consider the time derivatives as small quantities and neglect the terms of the order $V^{2}$, the field equations are given by Hillebrandt and Steinmetz (1976) and Herrera and Santos (1997)

$$
\begin{gathered}
e^{-\lambda}\left(\frac{\lambda^{\prime}}{r}-\frac{1}{r^{2}}\right)+\frac{1}{r^{2}}=8 \pi G \rho^{(\mathrm{eff})}, \\
e^{-\lambda}\left(\frac{\nu^{\prime}}{r}+\frac{1}{r^{2}}\right)-\frac{1}{r^{2}}=8 \pi G p_{r}^{(\mathrm{eff})}, \\
\frac{1}{2} e^{-\lambda}\left(\nu^{\prime \prime}+\frac{\nu^{\prime 2}}{2}+\frac{\nu^{\prime}-\lambda^{\prime}}{r}-\frac{\nu^{\prime \prime}}{\lambda} 2\right) \\
-\frac{1}{2} e^{-\lambda} \ddot{\lambda}=8 \pi G p_{\perp}^{(\mathrm{eff})}, \\
e^{-\lambda / 2} \frac{\dot{\lambda}}{r}=8 \pi G\left[\rho^{(\mathrm{eff})}+p_{r}^{(\mathrm{eff})}\right] \dot{r},
\end{gathered}
$$

where a dot means the derivative with respect to $t$.

We perturb the equilibrium solution

$$
\begin{aligned}
\rho^{(\mathrm{eff})} & =\rho_{0}^{(\mathrm{eff})}+\delta \rho^{(\mathrm{eff})}, \\
p_{r}^{(\mathrm{eff})}=p_{0 r}^{(\mathrm{eff})}+\delta p_{r}^{(\mathrm{eff})} & , \\
p_{\perp}^{(\mathrm{eff})} & =p_{0 \perp}^{(\mathrm{eff})}+\delta p_{\perp}^{(\mathrm{eff})}, \\
\nu=\nu_{0}+\delta \nu, \quad \lambda & =\lambda_{0}+\delta \lambda,
\end{aligned}
$$

where the index 0 refers to the equilibrium quantities. By expanding Eqs. (22)-(25) to first order in the perturbations we obtain (Hillebrandt and Steinmetz 1976)

$$
\begin{gathered}
8 \pi G \delta p_{r}^{(\mathrm{eff})}=\frac{e^{-\lambda_{0}}}{r^{2}}\left[r\left(\delta \nu^{\prime}-\nu_{0} \delta \lambda^{\prime}\right)-\delta \lambda\right], \\
8 \pi G \delta p_{\perp}^{(\mathrm{eff})}=\frac{1}{2} r \frac{\partial \delta p_{r}^{(\mathrm{eff})}}{\partial r}+\delta p_{r}^{(\mathrm{eff})} \\
+\frac{1}{4} \nu_{0}^{\prime} r\left[\delta \rho^{(\mathrm{eff})}+\delta p_{r}^{(\mathrm{eff})}\right] \\
+\left[\rho_{0}^{(\mathrm{eff})}+p_{0 r}^{(\mathrm{eff})}\right]\left[\frac{1}{2} e^{\lambda_{0}} r \ddot{r}+\frac{1}{4} r \delta \nu^{\prime}\right], \\
8 \pi G \delta \rho^{(\mathrm{eff})}=\frac{1}{r^{2}} \frac{\partial}{\partial r}\left(r e^{-\lambda_{0}} \delta \lambda\right), \\
8 \pi G\left[\rho_{0}^{(\mathrm{eff})}+p_{0 r}^{(\mathrm{eff})}\right] r \dot{r}=-e^{-\lambda_{0}} \delta \dot{\lambda} .
\end{gathered}
$$

In the case of the Einstein cluster we have $p_{0 r}^{(\mathrm{eff})}=0$, and since the spherical symmetry of the perturbed system is preserved, we may also take $\delta p_{r}^{\text {(eff) }}=0$. By assuming 
that all the perturbations are due to a small perturbation in $r=r(r, t)=r_{0}+\delta r(r, t)$, and after introducing the ansatz $\delta r(r, t)=e^{i \omega t} \xi(r)$ and denoting $v(r)=e^{-i \omega t} r^{2} e^{-\nu / 2} \delta r(r, t)$, the equation governing the radial pulsations of the Einstein cluster reduces to the simple algebraic condition

$$
8 \pi G \rho_{0}^{(\mathrm{eff})} e^{\lambda_{0}-\nu_{0}}\left[\frac{e^{5 \nu_{0} / 2+\lambda_{0} / 2}}{r^{2}}-\omega^{2}\right] v(r)=0 .
$$

Since $v(r) \neq 0$, we obtain the condition

$$
\omega^{2}=e^{5 \nu_{0} / 2+\lambda_{0} / 2} / r^{2}>0, \quad \forall r \in[0, R] .
$$

Stability means that the eigenfrequency $\omega$ of the lowest mode is real and positive, $\omega^{2}>$ 0 (Hillebrandt and Steinmetz 1976), which taking into account the previous condition, is obviously satisfied for the Einstein cluster. We therefore can conclude that the Einstein cluster is stable against small radial perturbations (a similar result was obtained by Gilbert (1954) by using a different method). The frequency of the radial oscillations of the cluster is related to its radius $R$ and mass $M$ by the relation $\omega^{2}=(1-2 G M / R)^{2} / R^{2}$.

\subsection{Stability against non-radial perturbations}

For isotropic spherically symmetric systems the stability against radial pulsations implies the stability against all small adiabatic oscillations (Binney and Tremaine 1987; Persic et al. 1996). For anisotropic models this need not to be true since the anisotropy may serve as a source of instability once the exact spherical symmetry is broken (Hillebrandt and Steinmetz 1976). In the case of Einstein clusters the condition of the exact circular orbits implies the vanishing of the radial pressure. However, as far as this condition is broken, and the trajectories of the particles are not perfectly circular, a radial pressure may be generated, and this pressure, together with the tangential pressure, may destabilise the system. Therefore it is important to also analyse the stability of the Einstein cluster under non-radial perturbations. Since the general relativistic analysis of this problem is extremely complicated and analytic solutions are difficult to be obtained, we restrict ourselves to the Newtonian case and follow the analysis given by Hillebrandt and Steinmetz (1976).

We assume that the perturbed Einstein cluster with non-spherical symmetry has a density $\rho$, a radial pressure $p_{r}$ and a tangential pressure $p_{\perp}$, and that generally $p_{r} \neq p_{\perp}$. As a result of the perturbation, the Newtonian equations of motion of the perturbed system, moving with velocity $\vec{v}$ under the action of the hydrodynamic force $\vec{F}$ in a gravitational field with potential $\Phi$ will be the anisotropic Euler equation, the continuity equation and the Poisson equation, given by

$$
\rho \frac{d \vec{v}}{d t}=\vec{F}-\rho \nabla \Phi, \frac{\partial \rho}{\partial t}+\nabla \cdot(\rho \vec{v})=0
$$

and

$$
\nabla^{2} \Phi=4 \pi G \rho
$$

respectively. In spherical coordinates $(r, \theta, \varphi)$ the compo- nents of the hydrodynamical force $F$ are

$$
\begin{aligned}
& F_{r}=-\frac{\partial p_{r}}{\partial r}+\frac{2 \Delta}{r}, \\
& F_{\theta}=-\frac{1}{r} \frac{\partial p_{\perp}}{\partial \theta}, \quad F_{\varphi}=-\frac{1}{r \sin \theta} \frac{\partial p_{\perp}}{\partial \varphi} .
\end{aligned}
$$

To close the system of equations we need an equation of state of the radial pressure, which we assume to be of the barotropic form, $p_{r}=p_{r}(\rho)$, and an equation of state for the anisotropy parameter $\Delta$, which we take as $\Delta=\beta(\rho) p_{r}$, with $\beta(\rho)$ an arbitrary function. We also denote $\gamma=\left(\rho / p_{r}\right)\left(\partial p_{r} / \partial \rho\right)$. In the following we will assume only axially symmetric perturbations, so that $\delta \varphi=0$.

By assuming that $\delta \vec{r}(\vec{r}, t)=e^{i \sigma t} \delta \vec{r}(\vec{r})$, the linearised equations of motion become

$$
\begin{aligned}
& \sigma^{2} \delta r=\frac{\partial \delta \Phi}{\partial r}-\frac{\delta \rho}{\rho^{2}} \frac{\partial p_{r}}{\partial r}+\frac{1}{\rho} \frac{\partial \delta p_{r}}{\partial r} \\
&-\frac{2}{r \rho}\left(\delta \Delta-\frac{\delta r}{r} \Delta-\frac{\delta \rho}{\rho} \Delta\right) \\
& \sigma^{2} \delta \theta=\frac{1}{r} \frac{\partial}{\partial \theta}\left(\delta \Phi+\frac{\delta p_{\perp}}{\rho}\right) \\
& \frac{\delta \rho}{\rho}+\frac{\delta r}{r} \frac{\partial \rho}{\partial r}+\frac{1}{r^{2}} \frac{\partial}{\partial r}\left(r^{2} \delta r\right)+\frac{1}{r \sin \theta} \frac{\partial}{\partial \theta}(r \sin \theta \delta \theta)=0 \\
& \nabla^{2} \delta \Phi=4 \pi G \delta \rho
\end{aligned}
$$

respectively, where all the quantities without $\delta$ are equilibrium quantities. We now express all the perturbations in terms of the Legendre polynomials $P_{l}(\cos \theta)$, so that

$$
\delta \Phi=\delta \Phi^{*}(r) P_{l}(\cos \theta), \quad \delta \rho=\delta \rho^{*} P_{l}(\cos \theta), \quad \text { etc. }
$$

Eq. (39) thus becomes

$$
\begin{aligned}
\sigma^{2} \delta r^{*}=\frac{\partial \delta \Phi^{*}}{\partial r}- & \frac{\delta \rho^{*}}{\rho^{2}} \frac{\partial p_{r}}{\partial r}+\frac{1}{\rho} \frac{\partial \delta p_{r}^{*}}{\partial r} \\
& -\frac{2}{r \rho}\left(\delta \Delta^{*}-\frac{\delta r^{*}}{r} \Delta-\frac{\delta \rho^{*}}{\rho} \Delta\right),
\end{aligned}
$$

By introducing the new variables $\xi=\gamma p_{r} \delta \rho^{*} / \rho, \varepsilon=\delta \Phi^{*}$ and $\eta=r^{2} \delta r^{*}$ we obtain the non-radial perturbation equations for an anisotropic system as Hillebrandt and Steinmetz 1976)

$$
\begin{gathered}
\eta^{\prime}=-\frac{p_{r}^{\prime}}{\gamma p_{r}}+\frac{l(l+1)}{\sigma^{2}} \varepsilon \\
-\left\{\frac{r^{2}}{\gamma p_{r}}-\frac{l(l+1)}{\sigma^{2} \rho}\left[(\beta+1)+\frac{\rho}{\gamma}\left(\frac{\partial \beta}{\partial \rho}\right)\right]\right\} \xi, \\
\varepsilon^{\prime \prime}=4 \pi G \frac{\rho}{\gamma p_{r}} \xi-\frac{2}{r} \varepsilon^{\prime}+\frac{l(l+1)}{r^{2}} \varepsilon, \\
\xi^{\prime}=\left(\sigma^{2} \rho+\frac{2 \beta}{r^{2}} p_{r}\right) \frac{\eta}{r^{2}}-\rho \varepsilon^{\prime} \\
-\frac{2}{r} \rho\left[\frac{\beta}{\rho}\left(1-\frac{1}{\gamma}\right)+\frac{1}{\gamma}\left(\frac{\partial \beta}{\partial \rho}\right)+\frac{1}{\gamma \rho} \frac{p_{r}^{\prime}}{p_{r}}\right] \xi .
\end{gathered}
$$

Eqs. (45)- (47) must be solved together with the boundary conditions $\xi(0)=\eta(0)=\varepsilon(0)=0, \varepsilon$ continuous across the boundary $R$ and $\Delta p_{r}^{*}(R)=\delta p_{r}^{*}(r)+\left.p_{r}^{\prime} \delta r^{*}(r)\right|_{r=R}=$ 
0 . Any solution satisfying these conditions determines one eigenvalue $\sigma^{2}$ (Hillebrandt and Steinmetz 1976).

We consider now the Einstein cluster as our equilibrium model. Moreover, we will consider only quadrupole oscillations, thus taking $l=2$. Since in this case $p_{r}=0$, it immediately follows that $\xi=0$. Hence Eq. (46) becomes

$$
\varepsilon^{\prime \prime}+\frac{2}{r} \varepsilon^{\prime}-\frac{6}{r^{2}} \varepsilon=0,
$$

with the solution $\varepsilon(r)=C_{1} r^{2}$, with $C_{1}$ an arbitrary constant of integration. Eq. (47) gives $\varepsilon^{\prime}=\sigma^{2} \eta / r^{2}$, and after taking the derivative of Eq. (45) it follows that $\eta$ satisfies the second order differential equation

$$
\eta^{\prime \prime}-\frac{6}{r^{2}} \eta=0
$$

with the solution $\eta(r)=C_{2} r^{3}$. From the definition of $\eta$ we thus obtain $\delta r^{*}=C_{2} r$. The continuity of the gravitational potential at the boundary $r=R$ gives the ratio of the integration constants as $C_{1} / C_{2}=G M / R^{3}$. In the case of the Einstein cluster the condition $\Delta p_{r}^{*}(R)=0$ gives $\delta p_{r}^{*}(R)=0$.

For the Einstein cluster $\Delta \approx \rho V_{0}^{2} / 2$ and $V_{0}^{2} \approx G m(r) / r$, where $V_{0}=$ constant is the speed in the constant velocity region. By estimating Eq. (44) near $r=R$ gives

$$
\left.\sigma^{2}(r)\right|_{r=R}=2 G M / R^{3}+G m(r) /\left.r^{3}\right|_{r=R}>0 .
$$

Since $\sigma^{2}$ is always positive, it follows that the Einstein clusters are stable with respect to the quadrupole oscillations.

\section{PHYSICAL BOUNDS FOR THE EINSTEIN CLUSTERS}

In realistic physical models for general relativistic matter distributions, the anisotropy $\Delta$ should be finite, positive and should satisfy the dominant energy condition (DEC) $\Delta \leqslant \rho$ and the strong energy condition (SEC) $2 \Delta \leqslant \rho$, see e.g. Böhmer and Harko (2006). These conditions may be written together as $\Delta \leqslant n \rho$, where $n=1$ for DEC and $n=1 / 2$ for SEC, and they are automatically satisfied by the components of the energy-momentum tensor of the Einstein cluster.

If the function $\Delta e^{\nu / 2} / r$ is monotonically decreasing for all $r$, then, as was proved rigorously in Böhmer and Harko (2005b), inside the anisotropic matter distribution the following inequality always holds

$$
\sqrt{1-\frac{2 G m(r)}{r}-\frac{\Lambda}{3} r^{2}} \geqslant \frac{1}{3}\left(1-\frac{\Lambda}{\langle\rho\rangle}\right) \frac{1}{1+f},
$$

where $f=f(m, r, \Lambda, \Delta)$, and we have already inserted that $p_{r}=0$. The function $f$ explicitly reads

$$
f=2 \frac{\Delta(r)}{\langle\rho\rangle}\left\{\frac{\arcsin \sqrt{2 G \alpha(r) m(r) / r}}{\sqrt{2 G \alpha(r) m(r) / r}}-1\right\},
$$

where we have denoted

$$
\alpha(r)=1+\frac{\Lambda r^{3}}{6 G m(r)},
$$

and where $\langle\rho\rangle$ is the mean density of the matter $\langle\rho\rangle=$ $3 m(r) / 4 \pi r^{3}$ inside the radius $r$.
For small values of the argument, we can use the series expansion $\arcsin x / x \approx 1+x^{2} / 6+\ldots$, and obtain

$$
f \approx \frac{2}{3} \frac{\Delta(r)}{\langle\rho\rangle} \frac{G \alpha(r) m(r)}{r}
$$

Since for the Einstein cluster the function $\Delta e^{\nu / 2} / r=$ $1 / r^{3-V_{0}^{2}}$ is monotonically decreasing, the above bound can be applied to the constant velocity region. Let us estimate Eq. (51) at the boundary $r=R$ of the cluster and denote by $M=m(R)$ its total mass. By neglecting small terms $\left(\Lambda r^{2} \ll 1, \Lambda V_{0}^{2} \ll 1\right)$ the above bound reduces to

$$
\sqrt{1-\frac{2 G M}{R}} \geqslant \frac{1}{3}\left(1-\frac{\rho(R)}{3\langle\rho\rangle} V_{0}^{2} \frac{G M}{R}\right) .
$$

Both terms containing $M / R$ can be replaced by the rotational velocity in view of Eq. (19), where the cosmological constant is neglected again. Hence, in terms of $V_{0}$, the condition given by Eq. (55) can be reformulated as

$$
V_{0}^{2} \leqslant \frac{4}{9}\left(1+\frac{4}{9} \frac{\rho(R)}{27\left\langle\rho_{R}\right\rangle}\right)
$$

where $\left\langle\rho_{R}\right\rangle$ is the average density of the whole cluster. On the other hand, by using the definition of the mean density and Eqs. (19) and (21) we have

$$
\frac{\rho(R)}{\left\langle\rho_{R}\right\rangle}=\frac{1}{3}
$$

Hence, one obtains the following absolute bound for the rotational velocity of the massive particles in the Einstein cluster

$$
V_{0}^{2} \leqslant \frac{2}{3} \sqrt{1+\frac{2^{2}}{3^{6}}}
$$

Therefore, we find the condition $V_{0} \leqslant 2 / 3 \approx 2 \times 10^{8} \mathrm{~m} / \mathrm{s}$. This result is consistent with the bound obtained in Gilbert (1954) from stability considerations, requiring that the velocity of particles in stable circular orbits in Einstein clusters must be smaller than half of the speed of light. On the other hand, the Buchdahl limit for the mass-radius ratio for the Einstein cluster (insert $G M / R$ for $\left.V_{0}^{2}\right)$ ) gives no physically relevant restriction since for typical galaxies $M / R \ll 1$. The same analysis can in principle be repeated without neglecting the contributions due to the cosmological constant and the anisotropy in Eq. (51). However, the resulting bound on the tangential velocity is only mildly effected and the main result is unchanged.

Following the analysis of Böhmer and Harko (2006) it is natural to consider next the behaviour of the invariant curvature scalars $r_{0}=R, r_{1}=R_{a b} R^{a b}$ and $r_{2}=R_{a b c d} R^{a b c d}$ which are, in general, decreasing functions with respect to the radius for regular relativistic matter distributions. For the Einstein cluster, these invariants only yield the speed of light as an upper bound on the velocity and no further condition emerges.

In the presence of the cosmological constant, the Buchdahl inequality (51) not only leads to upper bounds on the mass, but also implies the existence of a minimal mass (Böhmer and Harko 2005b). For Einstein clusters with radii $R_{g}$ in the range $R_{g} \approx 10 \mathrm{kpc}-100 \mathrm{kpc}$ this yields

$$
M_{\min }=\frac{4 \pi}{3} \frac{\Lambda}{16 \pi G} R_{g}^{3} \approx 4.5 \times 10^{5}-4.5 \times 10^{8} M_{\odot},
$$

perfectly consistent with present mass estimates of galaxies. 
In fact, this minimal mass is roughly of the order of the total (virial) mass of the galaxies, and further supports that the minimal mass due to $\Lambda$ is an important physical quantity in an astrophysical context, see e.g. Balaguera-Antolinez et al. (2005, 2006) and Balaguera-Antolinez and Nowakowski (2005).

\section{LIGHT DEFLECTION AND LENSING BY EINSTEIN CLUSTERS}

One of the ways we could in principle test the possible existence of Einstein clusters as astrophysical systems would be by studying the light deflection in the cluster, and in particular by studying the deflection of photons passing through the region where the rotation curves are flat, with $V=V_{0}=$ constant.

The metric coefficient $\exp (\nu)$ can be found from Eq. (11), and is given by

$$
e^{\nu}=\left(\frac{r}{R_{c}}\right)^{2 V_{0}^{2}}
$$

where $R_{c}$ is a constant of integration. Its value can be found by matching the metric tensor at the boundary $r=R$ with the standard Schwarzschild-de Sitter metric. Thus we obtain

$$
e^{\nu}=\left(1-2 V_{0}^{2}-\frac{\Lambda}{3} R^{2}\right)\left(\frac{r}{R}\right)^{2 V_{0}^{2}}
$$

Let us consider a photon approaching a galaxy from far distance. The bending of light by the gravitational field results in a deflection angle $\Delta \phi$ given by

$$
\Delta \phi=2\left|\phi\left(r_{0}\right)-\phi_{\infty}\right|-\pi
$$

where $\phi_{\infty}$ is the incident direction and $r_{0}$ is the coordinate radius of the closest approach to the centre of the galaxy. Generally, one finds (Weinberg 1972)

$$
\phi\left(r_{0}\right)-\phi_{\infty}=\int_{r_{0}}^{\infty} \frac{e^{\lambda(r) / 2}}{\sqrt{e^{\nu\left(r_{0}\right)-\nu(r)}\left(\frac{r}{r_{0}}\right)^{2}-1}} \frac{d r}{r} .
$$

For the Einstein cluster in the constant velocity region we obtain

$$
\phi\left(r_{0}\right)-\phi_{\infty}=\frac{\int_{r_{0}}^{\infty}\left[\left(\frac{r}{r_{0}}\right)^{2\left(1-V_{0}^{2}\right)}-1\right]^{-1 / 2} d r / r}{\sqrt{1-2 V_{0}^{2}}} .
$$

By introducing the new variable $\eta=r / r_{0}$ this leads to

$$
\phi\left(r_{0}\right)-\phi_{\infty}=\frac{\int_{1}^{\infty}\left[\eta^{2\left(1-V_{0}^{2}\right)}-1\right]^{-1 / 2} d \eta / \eta}{\sqrt{1-2 V_{0}^{2}}}
$$

and this integral can be evaluated exactly. Thus, we find the deflection angle for the Einstein cluster as

$$
\Delta \phi_{E C}=\frac{2 \pi V_{0}^{2}}{\sqrt{1-2 V_{0}^{2}}\left(1-V_{0}^{2}\right)} \approx 2 \pi V_{0}^{2} .
$$

In the standard approach to dark matter lensing it is assumed that the deflection angle $\Delta \phi_{D M}$ is given by $\Delta \phi_{D M}=4 G M(r) / r$, where $M(r)$ is the mass inside a radius $r$ Wambsganss (1998). In the constant velocity region, the usual deflection angle therefore becomes

$$
\Delta \phi_{D M}=4 V_{0}^{2}
$$

which is differs from the Einstein cluster deflection angle by a factor of $\pi / 2$.

In the generic case of a three-dimensional mass distribution, the density $\rho(\vec{r})$ can be projected along the line of sight into the lens plane to obtain the two-dimensional surface mass density distribution $\Sigma(\vec{\xi})=\int_{0}^{D_{S}} \rho(\vec{r}) d z$, where $D_{S}$ is the distance from the source to the observer and $\vec{\xi}$ is a two-dimensional vector in the lens plane Wambsganss (1998).

If one assumes for the galaxy lenses the singular isothermal density sphere model, with density varying as $\rho(r)=$ $V_{0}^{2} / 4 \pi G r^{2}$, the circularly symmetric mass distribution is given by $\Sigma(\xi)=V_{0}^{2} / 4 G \xi$. Since $M(\xi)=\int_{0}^{\xi} \Sigma\left(\xi^{\prime}\right) 2 \pi \xi^{\prime} d \xi^{\prime}=$ $\pi V_{0}^{2} / 2 G \xi$, we find $\alpha_{D M}(\xi)=2 \pi V_{0}^{2}$, which is constant, that is, independent of the impact parameter $\xi$ Wambsganss 1998). In the case of the Einstein cluster we obtain

$$
\alpha_{E C}(\xi)=\frac{\pi(\pi / 2+1) V_{0}^{2}}{\sqrt{1-\pi V_{0}^{2}}\left(1-V_{0}^{2}\right)} \approx \pi\left(\frac{\pi}{2}+1\right) V_{0}^{2} .
$$

Therefore, lensing effects can in principle discriminate between two different dark models. For the Einstein cluster model in comparison with the isothermal dark matter halo, we find

$$
\frac{\alpha_{D M}}{\alpha_{E C}}=\frac{4}{\pi+2} \approx 0.77
$$

This suggest that a galactic dark matter halo consisting of weakly interacting massive particles in the form of an Einstein cluster predicts slightly smaller gravitational lensing effects. Thus, lensing seems to be a prime experimental tool to test different dark matter models since they in general predict different deflection angles.

\section{CONCLUSIONS}

The flattened galactic rotation curves and the absence of sufficient luminous matter to explain them continue to pose a challenge to present day astrophysics. One would like to have a better understanding of some of the phenomena associated with them, like their universality and the very good correlation between the amount of dark matter and the luminous matter in galaxies.

In the present paper we have further developed an alternative view to the dark matter problem proposed by Lake (2006), namely, the possibility that the dark matter is in the form of an Einstein cluster of WIMPs. This assumption can explain the observed linearly increasing mass profile outside galaxies, without introducing any supplementary conditions. In fact, this model can reproduce any velocity profile Lake (2006). We analysed the stability of the Einstein clusters against both radial and non-radial perturbations, and we showed that the system is dynamically stable.

The Buchdahl bounds for the clusters have also been obtained, and thus we showed that there is a maximal bound for the velocity of the particles, and a minimum mass for the Einstein cluster. This minimal mass is of the same order of as the total (virial) masses of the galaxies. The existence of the minimum mass is a direct consequence of the presence of a non-zero cosmological constant Böhmer and Harko (2005b).

All the relevant physical quantities have been expressed in terms of observable parameters (mass, radius and velocity dispersion). This allows an in depth comparison of the 
predictions of the Einstein cluster model with observational results.

A possibility of observationally testing the viability of the Einstein cluster as a dark matter model is via gravitational lensing. We studied the lensing effect for Einstein clusters, and compared it with the standard dark matter model, the singular isothermal sphere. Even though the differences in the deflection angles are small, a significant improvement in the observational techniques may allow to discriminate

between the Einstein cluster and other dark matter models.

\section{ACKNOWLEDGEMENTS}

We would like to thank Roy Maartens for his valuable comments. The work of CGB was supported by research grant BO 2530/1-1 of the German Research Foundation (DFG). The work of $\mathrm{TH}$ is supported by the RGC grant No. 7027/06P of the government of the Hong Kong SAR.

\section{REFERENCES}

Balaguera-Antolinez A., Böhmer C.G., Nowakowski M., 2005, IJMP, D14, 1507

Balaguera-Antolinez A., Nowakowski M., 2005, A\&AS, 441, 23

Balaguera-Antolinez A., Böhmer C.G., Nowakowski M., 2006, CQG, 23, 485

Banerjee A., Som, M.M., 1981, Prog. Theor. Phys., 65, 1281

Binney, J., Tremaine, S., 1987, Galactic dynamics, Princeton University Press, Princeton

Böhmer C.G., Harko T., 2005a, PRD, 71, 084026

Böhmer C.G., Harko T., 2005b, PLB, 630, 73

Böhmer C.G., Harko T., 2006, CQG, 23, 6479

Comer G.L., Katz J., 1993, CQG, 10, 1751

Comer G.L., Langlois D., Peter P., 1993, CQG, 10, L127

Einstein A., 1939, Annals Math., 40, 922

Gilbert C., 1954, MNRAS, 114, 628

Herrera, L., Santos, N.O., 1997, Phys. Repts., 286, 53

Hillebrandt, W., Steinmetz, K.O., 1976, A\&A, 53, 283.

Hledik, S., Stuchlik, Z., Mrazova,K., gr-qc/0701051

Hogan P., 1973, Proc. Royal Irish Acad., Sect. A, 73, 91

Lake K., 2006, gr-qc/0607057

Landau L.D., Lifshitz E.M., 1998, The classical theory of fields, Butterworth-Heinemann

Persic, M.,Salucci, P., Stel, F., 1996, MNRAS, 281, 27

Wambsganss J., 1998, Living Rev. Rel., 1, 12

Weinberg S., 1972, Gravitation and Cosmology : Principles and Applications of the General Theory of Relativity, Wiley \& Sons, New York 